\title{
ON STRICTLY MINIMAL TOPOLOGICAL DIVISION RINGS
}

\section{LEOPOLDO NACHBIN}

1. Introduction. It is well known that, for every real or complex topological vector space, the following statements hold good:

(1) A nonvanishing linear functional on the space is continuous if and only if its kernel is closed in the space;

(2) Every finite-dimensional space possesses only one admissible topology.

In this note we shall determine the widest classes of topological division rings that can be used as scalar domains of topological vector spaces so as to preserve these important propositions. The results thus obtained generalize part of the recent work of J. Braconnier [2] and I. Kaplansky [5]. ${ }^{1}$ In a subsequent paper we shall apply these results to other related questions.

A topological ring is a ring endowed with an admissible topology, that is, a Hausdorff topology on the ring with respect to which the ring operations $x+y,-x, x y$ are continuous. A topological vector space is a vector space over a topological division ring endowed with an admissible topology, that is, a Hausdorff topology on the vector space with respect to which the vector space operations $x+y, \lambda x$ are continuous. Throughout this note we shall understand the notion of completeness and completion for these topological systems in the sense formulated by A. Weil [8] (see also Bourbaki [1]). $\mathfrak{I}_{1}$ and $\mathfrak{T}_{2}$ being two topologies on the same point set, we write $\mathfrak{I}_{1} \leqq \mathfrak{I}_{2}$ to denote that every set open according to $\mathfrak{I}_{1}$ must be open according to $\mathfrak{I}_{2}$; and $\mathfrak{I}_{1}<\mathfrak{I}_{2}$ if, in addition to this, $\mathfrak{I}_{1} \neq \mathfrak{I}_{2}$.

2. Strictly minimal rings. Let $K$ be a topological division ring and $\mathfrak{I}_{K}$ be its admissible topology. A topology $\mathfrak{T}$ on $K$ is said to be admissible with respect to $\mathfrak{T}_{K}$ if $K$ endowed with $\mathfrak{I}$ is a topological vector space over $K$ endowed with $\mathfrak{I}_{K}$ : this means that the mapping $(x, y) \rightarrow x+y$ is continuous from $\mathfrak{I} \times \mathfrak{I}$ to $\mathfrak{T}$, and the mapping $(x, y)$ $\rightarrow x y$ is continuous from $\mathfrak{I}_{K} \times \mathfrak{T}$ to $\mathfrak{T}$. Putting $y=1$ in the last condition, we see that the identity mapping $x \rightarrow x$ is continuous from $\mathfrak{I}_{K}$ to $\mathfrak{T}$, that is, $\mathfrak{I} \leqq \mathfrak{I}_{K}$. An obvious partial converse to this fact is the following: if $\mathfrak{I}$ is an admissible topology on $K$ and $\mathfrak{I} \leqq \mathfrak{I}_{K}$, then $\mathfrak{I}$ is admissible with respect to $\mathfrak{I}_{K}$.

Presented to the Society, October 30, 1948; received by the editors August 13, 1948.

${ }^{1}$ Numbers in brackets refer to the bibliography at the end of the paper. 
A topological division ring $K$ is said to be minimal if its topology $\mathfrak{I}_{K}$ is a minimal element in the ordered set of all admissible topologies on $K$; that is, if there exists no admissible topology $\mathfrak{I}$ on $K$ such that $\mathfrak{I}<\mathfrak{I}_{K} .^{2}$ The topological division ring is said to be strictly minimal if there exists no topology $\mathfrak{I}$ on $K$ admissible with respect to $\mathfrak{I}_{K}$ such that $\mathfrak{I}<\mathfrak{I}_{K}$; it amounts to the same to say that the only topology on $K$ admissible with respect to $\mathfrak{I}_{K}$ is $\mathfrak{I}_{K}$ itself.

THEOREM 1. Every strictly minimal topological division ring is minimal.

Proof. Let $\mathfrak{I}_{K}$ be the admissible topology given on the division ring $K$, and let $\mathfrak{I}$ be another admissible topology on $K$. If $\mathfrak{I} \leqq \mathfrak{T}_{K}$ it follows that $\mathfrak{I}$ is admissible with respect to $\mathfrak{I}_{K}$; but $\mathfrak{I}_{K}$ is strictly minimal: therefore $\mathfrak{I}=\mathfrak{I}_{K}$, that is, $\mathfrak{I}_{K}$ is minimal.

Let $K$ be a topological division ring. $A$ set $A \subset K$ is said to be $l$-bounded if for any neighborhood $W$ of 0 there exists a neighborhood $V$ of 0 such that $V A \subset W$. If $K$ is discrete, every set $A$ is obviously bounded. Let us assume that $K$ is not discrete. If the set $A$ is bounded, for any neighborhood $W$ of 0 there exists some $\lambda \in K, \lambda \neq 0$, such that $\lambda A \subset W$ : it suffices to pick some $\lambda \neq 0$ in that neighborhood $V$ of 0 for which $V A \subset W$. Conversely, assume that corresponding to every neighborhood $W$ of 0 there exists $\lambda \in K$ with $\lambda \neq 0$ such that $\lambda A \subset W$. Given the neighborhood $W$ of 0 , take some neighborhood $W_{1}$ of 0 such that $W_{1} W_{1} \subset W$ and determine $\lambda \in K$ with $\lambda \neq 0$ such that $\lambda A \subset W_{1}$. Putting $V=W_{1} \lambda$, we have a neighborhood of 0 and $V A \subset W$ : therefore $A$ is $l$-bounded. The set $A$ is said to be restricted if $0 \notin \bar{B}$, where $B=(A-0)^{-1}$, where the bar denotes closure in $K$ and $A-0$ is the set of all $x \in A, x \neq 0$. It amounts to the same to say that there exists a neighborhood $V$ of 0 satisfying the following equivalent conditions: $1 \notin V A, 1 \notin A V$.

Every topological division ring has at least one restricted neighborhood of 0 . In fact, consider a neighborhood $W$ of 0 such that $1 \notin W$ and take a neighborhood $V$ of 0 such that $V V \subset W$ : then $1 \notin V V$ and therefore $V$ is restricted.

Every l-bounded set is restricted. Let $W$ be a neighborhood of 0 such

${ }^{2}$ A minimal admissible topology on $K$, even when $K$ is complete under it, need not be the first element of the ordered set of all admissible topologies on $K$. In fact, let $R$ be the field of real numbers and $\mathfrak{T}$ be the natural topology on $R$. It is known (see Dieudonne [3]) that there exists an admissible topology $\mathfrak{T}^{*}$ on $R$ such that the completion of $R$ under $\mathfrak{T}^{*}$ is isomorphic to the field of complex numbers endowed with its usual topology. By the remark af ter the proof of Theorem 2, we see that both $\mathfrak{I}$ and $\mathfrak{T}^{*}$ are minimal on $R$. But $\mathfrak{T} \neq \mathfrak{T}^{*}$ : therefore $\mathfrak{T} \leqq \mathfrak{T}^{*}$ is false. 
that $1 \notin W$. If the set $A \subset K$ is bounded, there exists some neighborhood $V$ of 0 such that $V A \subset W$ and therefore $1 \notin V A$.

THEOREM 2. Every nondiscrete topological division ring in which all restricted sets are l-bounded is strictly minimal.

Proof. Let $K$ be the given division ring and $\mathfrak{T}_{K}$ be its admissible topology. Consider a topology $\mathfrak{I}$ on $K$ admissible with respect to $\mathfrak{I}_{K}$. We have already seen that $\mathfrak{T} \leqq \mathfrak{I}_{K}$. Let $V_{0}$ be a neighborhood of 0 according to $\mathfrak{I}$ such that $1 \notin V_{0}$. The continuity of the mapping $(x, y) \rightarrow x y$ from $\mathfrak{I}_{K} \times \mathfrak{I}$ to $\mathfrak{I}$ implies the existence of a neighborhood $V_{1}$ of 0 according to $\mathfrak{I}_{K}$ and a neighborhood $V_{2}$ of 0 according to $\mathfrak{T}$ such that $V_{1} V_{2} \subset V_{0}$ and a fortiori $1 \notin V_{1} V_{2}$. This shows that $V_{2}$ is restricted according to $\mathfrak{I}_{K}$. By the assumption, $V_{2}$ is $l$-bounded according to $\mathfrak{I}_{K}$ and therefore corresponding to every neighborhood $V$ of 0 according to $\mathfrak{T}_{K}$ there exists some $\lambda \in K, \lambda \neq 0$, such that $\lambda V_{2} \subset V$. Since $\lambda V_{2}$ is a neighborhood of 0 according to $\mathfrak{T}$, we may infer that the same is true for $V$. This shows that $\mathfrak{I}_{K} \leqq \mathfrak{T}$ and finally $\mathfrak{I}=\mathfrak{I}_{K}$.

From the result just established, we may conclude that every nondiscrete topological division ring which admits a valuation preserving the topology (see Kaplansky [5]) is strictly minimal. More particularly, every nondiscrete locally compact division ring is strictly minimal.

3. One-dimensional spaces. At this moment, let us recall the usual notion of direct image of a topology in the vector space case. Let $E$ be a topological vector space over a topological division ring $K$, let $F$ be a vector space over $K$ and consider a linear transformation $\phi: E \rightarrow F$ from $E$ onto $F$, that is $\phi(E)=F$. Let $\mathfrak{I}_{E}$ and $\mathfrak{T}_{K}$ be the topologies given on $E$ and $K$, respectively, and define a topology $\mathfrak{I}_{F}$ on $F$ in the following way: a set $Y \subset F$ is said to be open according to $\mathfrak{T}_{F}$ if its inverse image $\phi^{-1}(Y)$ is open according to $\mathfrak{T}_{E}$. It is easy to see that: (1) $\mathfrak{I}_{F}$ is really a topology on $F,(2) \phi(X)$ is open according to $\mathfrak{I}_{F}$ for any $X$ open according to $\mathfrak{I}_{E},(3)$ the vector space operations on $F$ are continuous with respect to $\mathfrak{I}_{F}$ and $\mathfrak{I}_{K},(4) \mathfrak{I}_{F}$ is a Hausdorff topology if and only if the kernel $\phi^{-1}(0)$ is closed according to $\mathfrak{T}_{E}$, and finally $(5) \phi$ is continuous from $\mathfrak{I}_{E}$ to $\mathfrak{T}_{F}$. We call $\mathfrak{I}_{F}$ the direct image of $\mathfrak{I}_{E}$ under $\phi$.

Theorem 3. Let $K$ be a given topological division ring. Consider two topological vector spaces $E$ and $F$ over $K$, where $F$ is one-dimensional, and a nonvanishing linear transformation $\phi: E \rightarrow F$. If $K$ is strictly minimal, then $\phi$ is continuous if and only if its kernel $\phi^{-1}(0)$ is closed in $E$. If $K$ is not strictly minimal, this result need not be always true. 
Proof. If $\phi$ is continuous, it is obvious that its kernel must be closed in $E$ : this half of the proof does not depend on $K$. Conversely, let us assume that $K$ is strictly minimal and let $\mathfrak{I}_{K}$ be its topology. Assume also that $\phi^{-1}(0)$ is closed in $E$. Noticing that $\phi(E)=F$, we may introduce on $F$ the direct image $\mathfrak{I}_{F}$ of the topology $\mathfrak{I}_{E}$ given on $E$, and $\mathfrak{T}_{F}$ will be admissible on $F$. Since every one-dimensional vector space $F$ over $K$ is algebraically isomorphic to $K$ considered as a vector space over $K$, the assumption that $K$ is strictly minimal is equivalent to saying that every one-dimensional vector space over $K$ possesses only one admissible topology. Therefore $\mathfrak{T}_{F}$ coincides with the admissible topology previously given on $F$ and the proof is achieved by remarking that $\phi$ is continuous from $\mathfrak{I}_{E}$ to $\mathfrak{I}_{F}$. Now, let us assume that $K$ is not strictly minimal and let $\mathfrak{I}$ be a topology on $K$ admissible with respect to $\mathfrak{T}_{K}$ such that $\mathfrak{I}<\mathfrak{T}_{K}$. Consider the set $E=K$ endowed with $\mathfrak{I}$ as a topological vector space over $K$ endowed with $\mathfrak{I}_{K}$ and the set $F=K$ endowed with $\mathfrak{T}_{K}$ as a topological vector space over $K$ endowed with $\mathfrak{I}_{K}$. The nonvanishing linear transformation $\phi: E \rightarrow F$ defined by $\phi(x)=x$ for any $x \in E$ has a kernel $\phi^{-1}(0)=0$ closed in $E$, but $\phi$ is not continuous.

THEOREM 4. Let $E=E_{1} \oplus E_{2}$ be a topological vector space over a strictly minimal topological division ring $K$, where $E_{1}$ and $E_{2}$ are vector subspaces of $E$, the first being one-dimensional. Let $\mathfrak{T}$ be the admissible topology given on $E$ and $\mathfrak{I}_{1}$ and $\mathfrak{I}_{2}$ be the admissible topologies induced by $\mathfrak{T}$ on $E_{1}$ and $E_{2}$, respectively. Then we have $\mathfrak{T}=\mathfrak{I}_{1} \oplus \mathfrak{I}_{2}$ if and only if $E_{2}$ is closed in $E$.

Proof. It is clear that the relation $\mathfrak{T}=\mathfrak{I}_{1} \oplus \mathfrak{I}_{2}$ implies that $E_{2}$ (and also $E_{1}$ ) is closed in $E$. Conversely, let us assume that $E_{2}$ is closed in $E$. Putting $\mathfrak{I}^{*}=\mathfrak{I}_{1} \oplus \mathfrak{T}_{2}$ we obtain an admissible topology on $E$. Consider any neighborhood $W$ of 0 in $E$ according to $\mathfrak{T}$ and determine some neighborhood $V$ of 0 in $E$ according to $\mathfrak{I}$ such that $V+V \subset W$. Then $W_{1}=V \cap E_{1}$ is a neighborhood of 0 in $E_{1}$ according to $\mathfrak{I}_{1}$ and $W_{2}=V \cap E_{2}$ is a neighborhood of 0 in $E_{2}$ according to $\mathfrak{I}_{2}$ and we have $W_{1}+W_{2} \subset W$. Since $W_{1}+W_{2}$ is a neighborhood of 0 in $E$ according to $\mathfrak{I}^{*}$, the same is true for $W$ and we have proved that $\mathfrak{I} \leqq \mathfrak{I}^{*}$. This half of the proof does not depend on the nature of $K$. On the other hand, every $x \in E$ may be expressed uniquely as $x=x_{1}+x_{2}$, where $x_{1} \in E_{1}$ and $x_{2} \in E_{2}$. Define $\pi_{1}: E \rightarrow E_{1}$ and $\pi_{2}: E \rightarrow E_{2}$ by $\pi_{1}(x)=x_{1}$ and $\pi_{2}(x)=x_{2}$. Since $E_{2}=\pi_{1}^{-1}(0)$ is closed in $E$, we may make use of Theorem 3 and say that $\pi_{1}: E \rightarrow E_{1}$ is continuous from $\mathfrak{I}$ to $\mathfrak{T}_{1}$. It amounts to the same to say that $\pi_{1}: E \rightarrow E$ is continuous from $\mathfrak{T}$ to $\mathfrak{T}$. Since $\pi_{2}(x)=x-\pi_{1}(x)$, we may also say that $\pi_{2}: E \rightarrow E$ is continuous 
from $\mathfrak{I}$ to $\mathfrak{I}$ or, equivalently, that $\pi_{2}: E \rightarrow E_{2}$ is continuous from $\mathfrak{I}$ to $\mathfrak{I}_{2}$. Having proved this, let there be given any neighborhood $W$ of 0 in $E$ according to $\mathfrak{T}^{*}$; by definition there exists a neighborhood $W_{1}$ of 0 in $E_{1}$ according to $\mathfrak{I}_{1}$ and also a neighborhood $W_{2}$ of 0 in $E_{2}$ according to $\mathfrak{T}_{2}$ such that $W_{1}+W_{2} \subset W$. By the continuity of $\pi_{1}$, we may find a neighborhood $V_{1}$ of 0 in $E$ according to $\mathfrak{I}$ such that $\pi_{1}\left(V_{1}\right) \subset W_{1}$, and similarly there exists a neighborhood $V_{2}$ of 0 in $E$ according to $\mathfrak{I}$ for which $\pi_{2}\left(V_{2}\right) \subset W_{2}$. Putting $V=V_{1} \cap V_{2}$, we see that $V \subset W_{1}+W_{2} \subset W$; therefore $W$ is a neighborhood of 0 in $E$ according to $\mathfrak{I}$ and we have proved that $\mathfrak{I}^{*} \leqq \mathfrak{T}$. In conclusion we have $\mathfrak{T}=\mathfrak{T}^{*}$.

$E$ and $F$ being two sets and $\phi: E \rightarrow F$ being a function from $E$ to $F$, the set $G(\phi)$ of all points $\{x, \phi(x)\}$ of the product space $E \times F$ will be called the graph of the function.

Theorem 5. Let $K$ be a given topological division ring. Consider two topological vector spaces $E$ and $F$ over $K$, where $F$ is one-dimensional, and a linear transformation $\phi: E \rightarrow F$. If $K$ is strictly minimal, then $\phi$ is continuous if and only if its graph $G(\phi)$ is closed in $E \times F$. If $K$ is not strictly minimal, this result need not be always true.

Proof. It is clear that the graph of a continuous function into a Hausdorff space is closed. Therefore the continuity of $\phi$ implies that $G(\phi)$ is closed. Conversely assume that $K$ is strictly minimal and $G(\phi)$ is closed. Define $\varphi(x, y)=\phi(x)-y$ for $x \in E, y \in F$. Then $\varphi: E$ $\times F \rightarrow F$ is a linear transformation with a closed kernel $\varphi^{-1}(0)=G(\phi)$. By Theorem 3, $\varphi$ is continuous and since $\varphi(x, 0)=\phi(x)$ we conclude that $\phi$ is continuous. The final part of the statement is proved exactly as in the case of Theorem 3 .

4. Finite-dimensional spaces. A partial extension of Tychonoff's theorem about the uniqueness of admissible topologies is the following:

THEOREM 6. Let $K$ be a given topological division ring. If $K$ is strictly minimal, every n-dimensional vector space $E$ over $K$ possesses only one admissible topology with respect to which all $n-1$ dimensional vector subspaces are closed. If $K$ is not strictly minimal this result need not be true.

Proof. Let $\mathfrak{T}$ be the admissible topology given on $E$. Since every vector subspace of $E$ is an intersection of $n-1$ dimensional vector subspaces, it follows from the assumptions that all vector subspaces of $E$ are closed. Let $E=E_{1} \oplus \cdots \oplus E_{n}$, each $E_{i}$ being a one-dimensional vector subspace of $E$. Put $E^{i}=E_{i} \oplus \cdots \oplus E_{n}$. Let $\mathfrak{T}_{i}$ and $\mathfrak{T}^{i}$ 
be the topologies induced by $\mathfrak{I}$ on $E_{i}$ and on $E^{i}$ respectively. Since $E^{2}$ is closed in $E$, we have $\mathfrak{T}=\mathfrak{I}_{1} \oplus \mathfrak{T}^{2}$ by Theorem 4 . Since $E^{3}$ is closed in $E$ and $E^{3} C E^{2}$, we see that $E^{3}$ is closed in $E^{2}$. Therefore $\mathfrak{T}^{2}=\mathfrak{T}_{2} \oplus \mathfrak{T}^{3}$ again by Theorem 4 , and so on. By induction we get $\mathfrak{T}=\mathfrak{T}_{1} \oplus \ldots$ $\oplus \mathfrak{I}_{n}$. Since $K$ is strictly minimal, each $\mathfrak{I}_{i}$ is the unique admissible topology on $E_{i}$; this implies the uniqueness of $\mathfrak{T}$. The final part of the statement is proved exactly as in the case of Theorem 3.

In the preceding theorem, it would be sufficient to assume the existence of $n$ closed vector subspaces $S_{1}, \cdots, S_{n}$ of dimension $n-1$ such that $S_{1} \cap \cdots \cap S_{n}=0$. In fact, let $S$ be another vector subspace of dimension $n-1$. Consider $n+1$ linear functionals $\phi_{i}: E \rightarrow K$ and $\phi: E \rightarrow K$ with kernels $S_{i}$ and $S(i=1, \cdots, n)$ respectively. Then there are $c_{1}, \cdots, c_{n} \in K$ such that $\phi(x)=c_{1} \phi_{1}(x)+\cdots+c_{n} \phi_{n}(x)$ for any $x \in E$. Since $S_{i}$ is closed, we see by Theorem 3 that each $\phi_{i}$ is continuous; therefore $\phi$ is continuous and $S$ is closed.

The complete extension of Tychonoff's theorem is as follows:

THEOREM 7. Let $K$ be a given topological division ring. In order that every finite-dimensional vector space over $K$ should have only one admissible topology it is necessary and sufficient that $K$ be strictly minimal and complete.

Proof. We have already remarked that every one-dimensional vector space over $K$ possesses only one admissible topology if and only if $K$ is strictly minimal. Let us assume that $K$ is not complete and consider its completion $\tilde{K}$, that is, the essentially unique complete topological ring containing $K$ as a dense topological subring (see Bourbaki [1]). By the assumption, we may pick some $\zeta \in \widetilde{K}-K$. Let $E \subset \tilde{K}$ be the set of all points $x \zeta+y$, where $x, y \in K$. Since $K \subset \tilde{K}$, we may say that $\tilde{K}$ is a topological vector space over $K$. But $E$ is a vector subspace of $\tilde{K}$. Therefore, the topology $\mathfrak{I}_{1}$ induced on the vector space $E$ by the topology of $\tilde{K}$ is admissible. Moreover $K \subset E$, $\bar{K}=E$ (the bar denoting closure in $E$ according to $\mathfrak{T}_{1}$ ) because $K$ is dense in $\tilde{K}$ and a fortiori in $E$. On the other hand, the mapping $(x, y) \rightarrow x \zeta+y$ is a vector space isomorphism between $K \times K$ and $E$. Since $K \times K$ is a topological vector space over $K$, we may transfer its topology to an admissible topology $\mathfrak{T}_{2}$ on $E$ and it is clear that $\bar{K}=K$ (where the bar now denotes closure in $E$ according to $\mathfrak{I}_{2}$ ). By this procedure, we have been able to set up two distinct admissible topologies $\mathfrak{I}_{1}$ and $\mathfrak{I}_{2}$ on the two-dimensional vector space $E$ over $K$. Therefore, if every two-dimensional vector space over $K$ must have a unique admissible topology, $K$ is to be complete. Conversely, let us assume that $K$ is strictly minimal and complete. This implies that 
every one-dimensional vector space over $K$ possesses only one admissible topology and, in addition, is complete under this topology. Assume that every $n-1$ dimensional vector space over $K$ possesses only one admissible topology and, in addition, is complete under this topology. Let us consider a vector space $E$ of dimension $n$ over $K$ and an admissible topology $\mathfrak{T}$ on $E$. For any $n-1$ dimensional vector subspace of $E$, the induced topology is its unique admissible topology and the subspace is complete under it; a fortiori the subspace is closed in $E$. By Theorem 6 it follows that $\mathfrak{I}$ is uniquely determined; in addition to this, we may infer from $\mathfrak{T}=\mathfrak{I}_{1} \oplus \cdots \oplus \mathfrak{I}_{n}$ (in the notation of the proof of Theorem 6 ) that $E$ is complete under $\mathfrak{T}$. The induction is thus completed.

THEOREM 8. Let $K$ be a given topological division ring. In order that every automorphism of any finite-dimensional topological vector space over $K$ should be continuous it is necessary and sufficient that $K$ be strictly minimal and complete.

Proof. Assume first that every automorphism is continuous. Let $\mathfrak{T}_{1}$ and $\mathfrak{I}_{2}$ be two admissible topologies on the vector space $K^{n}=K \times \cdots$ $\times K$ ( $n$ times). Consider the vector space $K^{n} \times K^{n}$ endowed with the admissible topology $\mathfrak{I}_{1} \times \mathfrak{I}_{2}$. Since $K^{n} \times K^{n}$ is a square of a set, the symmetry $\{x, y\} \rightarrow\{y, x\}$ has a meaning (where $x, y \in K^{n}$ ). This transformation is an automorphism of $K^{n} \times K^{n}$. By the hypothesis it is continuous and this amounts to saying that $\mathfrak{I}_{1}=\mathfrak{I}_{2}$. This shows that every finite-dimensional vector space over $K$ has a unique admissible topology. Applying the preceding theorem we see that $K$ is strictly minimal and complete. Conversely, if $K$ satisfies these conditions, we see by the same theorem that we have uniqueness of admissible topology in the finite-dimensional case: since the transform of an admissible topology under an algebraic isomorphism between two topological vector spaces is clearly admissible, we may infer that this algebraic isomorphism is also homeomorphic. More generally, if $\phi: E \rightarrow F$ is a linear transformation, with kernel $S=\phi^{-1}(0)$, between two finite-dimensional topological vector spaces, we may consider it as the product of the natural homomorphism $E \rightarrow E / S$ and the natural isomorphism $E / S \rightarrow \phi(E)$ : therefore $\phi$ is continuous.

Theorem 9. Let $K$ be a given topological division ring. Consider two topological vector spaces $E$ and $F$ over $K$, where $F$ is finite-dimensional, and a linear transformation $\phi: E \rightarrow F$ from $E$ onto $F$. If $K$ is strictly minimal and complete, then $\phi$ is continuous if and only if its kernel $\phi^{-1}(0)$ is closed in $E$. If $K$ is not strictly minimal, or is not complete, this result need not be always true. 
Proof. The sufficiency of the assumptions is proved exactly as in the case of Theorem 3, but now with the help of the preceding theorem. If the assumptions concerning $K$ are not fulfilled, we are able to find a vector space $E$ over $K$ of dimension one or two (see the first half of the proof of Theorem 7) endowed with two admissible topologies $\mathfrak{I}_{1}$ and $\mathfrak{I}_{2}$ such that $\mathfrak{I}_{1}<\mathfrak{I}_{2}$ : then the identity transformation of $E$ is not continuous from $\mathfrak{T}_{1}$ to $\mathfrak{T}_{2}$ but has a closed kernel.

THEOREM 10. Let $E=E_{1} \oplus E_{2}$ be a topological vector space over $a$ strictly minimal complete topological division ring $K$, where $E_{1}$ and $E_{2}$ are vector subspaces of $E$, the first being finite-dimensional. Let $\mathfrak{T}$ be the admissible topology given on $E$ and $\mathfrak{T}_{1}$ and $\mathfrak{T}_{2}$ be the admissible topologies induced by $\mathfrak{T}$ on $E_{1}$ and $E_{2}$, respectively. Then we have $\mathfrak{T}=\mathfrak{I}_{1} \oplus \mathfrak{I}_{2}$ if and only if $E_{2}$ is closed in $E$.

Proof. The proof is exactly the same as that given for Theorem 4, but now with the help of the preceding theorem.

The preceding Theorem 10 can be reformulated in the customary fashion as a result about the continuity of a projection with closed kernel of a topological vector space onto a finite-dimensional vector subspace, or a projection with finite-dimensional kernel of a topological vector space onto a closed vector subspace; similarly for Theorem 4 and projections with one-dimensional ranges or kernels.

TheOREM 11. Let $K$ be a given topological division ring. Consider two topological vector spaces $E$ and $F$ over $K$, where $F$ is finite-dimensional, and a linear transformation $\phi: E \rightarrow F$. If $K$ is strictly minimal and complete, then $\phi$ is continuous if and only if its graph is closed in $E \times F$. If $K$ is not strictly minimal, or is not complete, this resull need not be always true.

ProOF. The first part is proved as in the case of Theorem 5 by using Theorem 9. The final part is proved as in Theorem 9.

A topological vector space $E$ is said to be absolutely closed if $E$ is closed in every topological vector space containing it as a topological vector subspace. It is known that this is equivalent to saying that $E$ is complete. From Theorem 7 if follows that every finite-dimensional topological vector space over a strictly minimal complete topological division ring is complete. In different words, every finite-dimensional vector subspace of a topological vector space over a strictly minimal complete topological division ring is closed. A stronger result is the following:

THEOREM 12. Let $E$ be a topological vector space over a strictly minimal complete topological division ring $K$. If $E_{1}$ and $E_{2}$ are vector subspaces 
of $E$, the first being finite-dimensional and the second closed in $E$, then $E_{1}+E_{2}$ is a closed vector subspace of $E$.

Proof. Without loss of generality, we may assume that $E_{1}$ and $E_{2}$ are disjoint vector subspaces and therefore $E_{1}+E_{2}=E_{1} \oplus E_{2}$. Consider the completion $\tilde{E}$ of $E$, that is, the essentially unique complete topological vector space over $K$ containing $E$ as a dense topological vector subspace. Since $E_{1}$ is disjoint from $E_{2}$, we have $E_{1}-0 \subset E-E_{2}$. But $E-E_{2}$ is open in $E$ and this enables us to find a set $A \subset \tilde{E}$ open in $\tilde{E}$ such that $A \cap E=E-E_{2}$. Then $E_{1}-0 \subset A, E_{2} \subset \tilde{E}-A$ and therefore $\bar{E}_{2} \subset \tilde{E}-A$ (here and in the sequel the bar denotes closure in $\tilde{E}$ ). Thus $E_{1}-0 \subset \tilde{E}-\bar{E}_{2}$ and $E_{1}$ and $\bar{E}_{2}$ are disjoint vector subspaces of $\tilde{E}$. Let us prove the relation $\bar{F}=E_{1} \oplus \bar{E}_{2}$, where $F=E_{1} \oplus E_{2}$. The inclusion $\supset$ is obvious. By Theorem 10, the topology on $E_{1} \oplus \bar{E}_{2}$ is the direct sum of the topologies on $E_{1}$ and on $\bar{E}_{2}$. But $E_{1}$, being finitedimensional, is complete and $\bar{E}_{2}$, being closed in $\tilde{E}$, is also complete. This shows that $E_{1} \oplus \bar{E}_{2}$ is complete and thus closed in $\tilde{E}$. From this we conclude that the inclusion $C$ is true. Finally observing that $E_{1} \subset E$ and making use of the modularity law we have $E \cap \bar{F}=E$ $\cap\left(E_{1} \oplus \bar{E}_{2}\right)=E_{1} \oplus\left(E \cap \bar{E}_{2}\right)=E_{1} \oplus E_{2}$, that is, $E_{1} \oplus E_{2}$ is closed in $E$.

Theorem 12 was proved by Mackey (see [6, Lemma 2.1]) in the case of normed spaces. It is known (see Stone [7, p. 21]) that this theorem need not be true even in separable Hilbert space if we assume merely that $E_{1}$ and $E_{2}$ are closed in $E$.

\section{BIBLIOGRAPHY}

1. N. Bourbaki, Eléments de mathématiques: Topologie gênérale, Actualités Scientifiques et Industrielles, nos. 858, 916, and 1045, Paris, 1940-1948.

2. J. Braconnier, Sur les espaces vectoriels localement compacts, C. R. Acad. Sci. Paris vol. 222 (1946) pp. 777-778.

3. J. Dieudonné, Sur les corps topologiques connexes, C. R. Acad. Sci. Paris vol. 221 (1945) pp. 396-398.

4. I. Kaplansky, Topological rings, Amer. J. Math. vol. 69 (1947) pp. 153-183.

5. - Topological methods in valuation theory, Duke Math. J. vol. 14 (1947) pp. 527-541.

6. G. W. Mackey, Isomorphisms of normed linear spaces, Ann. of Math. vol. 43 (1942) pp. 244-260.

7. M. H. Stone, Linear transformations in Hilbert spaces and their applications to analysis, Amer. Math. Soc. Colloquium Publications, vol. 15, New York, 1932.

8. A. Weil, Sur les espaces à structure uniforme et sur la topologie générale, Actualités Scientifiques et Industrielles, no. 551, Paris, 1938.

UNIVERSITY OF BRAZIL 